\title{
Achieving high efficiency laminated polymer solar cell with interfacial modified metallic electrode and pressure induced crystallization
}

\author{
Yongbo Yuan, ${ }^{1} \mathrm{Yu} \mathrm{Bi}^{1,2}$ and Jinsong Huang ${ }^{1,3, a)}$ \\ ${ }^{1}$ Department of Mechanical Engineering, University of Nebraska-Lincoln, Lincoln, Nebraska 68588, USA \\ ${ }^{2}$ Key Laboratory of Semiconductor Materials Science, Institute of Semiconductors, Chinese Academy \\ of Sciences, Beijing 100083, People's Republic of China \\ ${ }^{3}$ Nebraska Center for Materials and Nanoscience, Lincoln, Nebraska 68583, USA
}

(Received 5 December 2010; accepted 20 January 2011; published online 11 February 2011)

\begin{abstract}
We report efficient laminated organic photovoltaic device with efficiency approach the optimized device by regular method based on Poly(3-hexylthiophene-2,5-diyl) and [6,6]-phenyl-C61-butyric acid methyl ester (PCBM). The high efficiency is mainly attributed to the formation of a concrete polymer/metal interface mechanically and electrically by the use of electronic-glue, and using the highly conductive and flexible silver film as anode to reduce photovoltage loss and modifying its work function for efficiency hole extraction by ultraviolet/ozone treatment, and the pressure induced crystallization of PCBM. (C) 2011 American Institute of Physics. [doi:10.1063/1.3554377]
\end{abstract}

One critical issue in the roll-to-roll mass production of organic photovoltaics (OPVs) is how to form good mechanical and electronic contact at the polymer/electrode interface so that the devices maintain the high device power conversion efficiency (PCE) fabricated by other methods. Direct thermal lamination of organic films have been reported to form planar and bulk heterojunction OPVs, where the heterojunctions were formed by laminating the two different semiconductor films at a high pressure $(\sim \mathrm{MPa})$ and a high temperature above the glass transition temperature of polymers. ${ }^{1,2}$ This approach is limited by the high temperatures and high pressures, which inevitably changes the morphology of the conjugated polymers and always degrades the performance of the OPVs. Alternatively the lamination of thin metal films or metallic nanowire network onto the polymer blends has been reported to generate electrodes. ${ }^{2-4}$ The formation of the electrodes by these soft lamination processes avoids damaging the semiconducting polymers by the energetic metal atoms, which occurs in the metal deposition by thermal evaporation. ${ }^{2}$ However, the reported efficiencies of the laminated devices are lower than that of regular devices due to the nonconcrete polymer/metal interfaces and a variety of other reasons.

This paper reports laminated OPVs with a PCE reaching the same level of optimized regular devices with electrodes deposited by thermal evaporation. This is achieved by selecting a proper electrode material-silver ( $\mathrm{Ag}$ )-as the anode for lamination as well as having a better control of the work function of the anode layer by oxidization. It was also found that the lamination process promotes the crystallization of [6,6]-phenyl-C61-butyric acid methyl ester (PCBM), contributing the observed high PCE of the laminated devices.

For the device fabrication, an ultrathin cesium carbonates $\left(\mathrm{Cs}_{2} \mathrm{CO}_{3}\right)$ layer was spin-coated onto the indium tin oxide (ITO) to form cathode. ${ }^{5,6}$ The thickness of succedent poly(3-hexylthiophene-2,5-diyl) (P3HT): PCBM (1:1 by wt) polymer layer was $180 \mathrm{~nm}$. On the anode side, $100 \mathrm{~nm} \mathrm{Ag}$ layer or Al layer was deposited on the poly(ethylene naph-

\footnotetext{
${ }^{\text {a) }}$ Author to whom correspondence should be addressed. Electronic mail: jhuang2@unl.edu.
}

thalate) substrate (PEN, Teonex Q65F,). The conducting polymer Poly(3,4-ethylenedioxythiophene) poly(styrenesulfonate) (PEDOT:PSS) (PH1000) was doped with D-sorbitol and employed as the Electronic glue (E-glue), ${ }^{5}$ which was spin-coated to form the adhesive anode. After drying both substrates, they were laminated together by a moderate pressure of $10-50 \mathrm{KPa}$ so that the two substrates were tightly glued together. The lamination process and device structure are illustrated in Fig. 1. The two half devices were laminated at a temperature of $100{ }^{\circ} \mathrm{C}$, which is a safe thermal annealing temperature for many conjugated polymers. The details of the lamination process have been reported previously. ${ }^{6}$

Several commonly used electrode materials in OPVs, such as Al, Ag, and ITO, were compared. To make a standard basis for the inverted OPVs, regular OPVs were fabricated with thermal deposited molybdenum oxide $\left(\mathrm{MoO}_{3}\right)$ (4 $\mathrm{nm}) / \mathrm{Ag}$ as the anode. The inverted OPVs with $\mathrm{MoO}_{3}$ as the anode buffer layer have been shown to have similar or superior performance compared to regular devices. ${ }^{7}$ Fig. 2(a) shows the current density-voltage $(I-V)$ curves of the OPVs with different laminated or evaporated anodes. Under the one sun simulated irradiation, the device with laminated E-glue/Ag anode shows the highest PCE compared to devices with laminated E-glue/ITO or E-glue/Al anode. The regular inverted OPV with $\mathrm{MoO}_{3} / \mathrm{Ag}$ anode shows a short

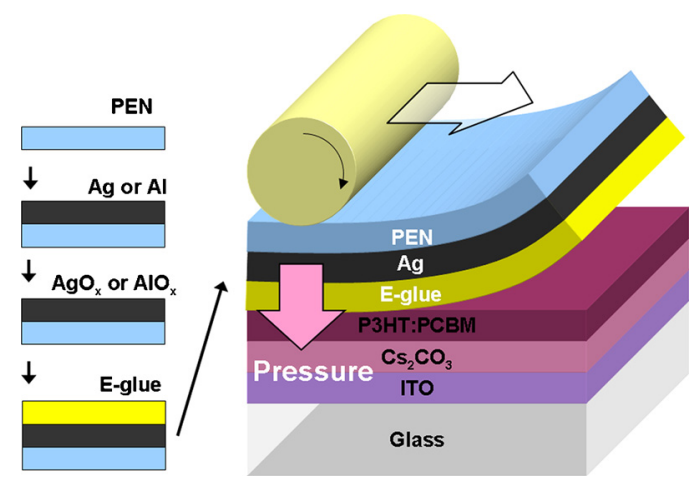

FIG. 1. (Color online) Schematic lamination process and the structure of inverted device. 

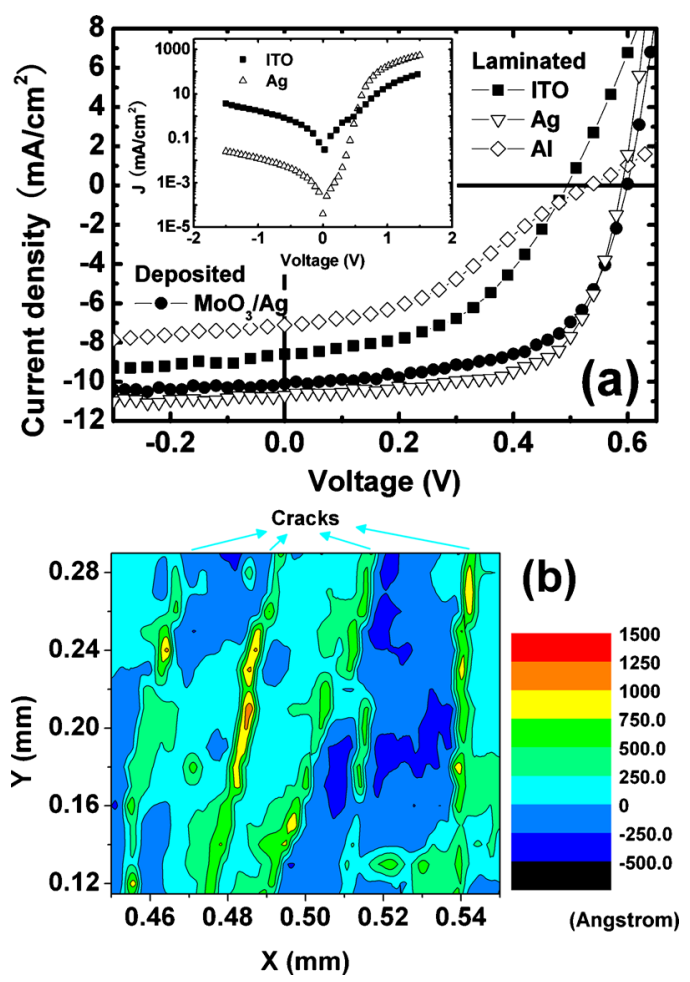

FIG. 2. (Color online) (a) Comparison of $I-V$ curve of inverted OPVs with different anodes. Inset shows the dark currents of laminated devices with ITO anode and Ag anode. (b) Surface profile of bended ITO on PEN flexible substrate.

circuit current density $\left(J_{\mathrm{sc}}\right)$ of $10.1 \mathrm{~mA} / \mathrm{cm}^{2}$, an open circuit voltage $\left(V_{\mathrm{oc}}\right)$ of $0.60 \mathrm{~V}$, a fill factor $(\mathrm{FF})$ of $60 \%$, and a PCE of $3.6 \%$. The device with laminated E-glue/ITO anode exhibits a $V_{\text {oc }}$ of $0.49 \mathrm{~V}$, a $J_{\text {sc }}$ of $8.6 \mathrm{~mA} / \mathrm{cm}^{2}$, a FF of $48 \%$, and a PCE of $2.1 \%$, which is consistent with our previous results. ${ }^{6}$ The device with E-glue/ $\mathrm{Ag}$ anode exhibits a $V_{\mathrm{oc}}$ of $0.59 \mathrm{~V}$, a $J_{\mathrm{sc}}$ of $10.7 \mathrm{~mA} / \mathrm{cm}^{2}$, a FF of $63 \%$, and a PCE of $4.0 \%$. This efficiency is the same with that of certified optimized devices. ${ }^{8}$

It is of interest to understand how this lamination process and material selection increased the device performance. The increase of $V_{\text {oc }}$ from 0.49 to $0.59 \mathrm{~V}$ can be partially explained by the reduced reverse dark saturation current. ${ }^{9-11}$ $V_{\text {oc }}$ variation due to the dark current change is roughly proportional to $(n k T / q) \ln \left(J_{\mathrm{ph}} / J_{s}\right)$, where $J_{\mathrm{ph}}$ is the photocurrent current, $J_{s}$ is the reverse dark saturation current, and $n$ is the ideality factor. As seen in the inset of Fig. 2(a), the leakage current of the device with E-glue/Ag anode is about 100 times lower than that of the laminated device with the ITO anode. In an ideal situation, the leakage current of the device with E-glue/ITO anode should be comparable to that of a device with E-glue/Ag anode. However, the fragile ITO layer on flexible PEN substrate easily forms cracks under bending during the lamination, wiping or rapid thermal treatment process. ${ }^{12}$ The surface profile mapping of the bent ITO on a PEN flexible substrate indicated a local high roughness of more than $100 \mathrm{~nm}$ [Fig. 2(b)]. The local high roughness on the surface results from the formation of cracks in ITO film. This was confirmed by testing the resistance of the ITO film during the bending process. The sheet resistance of the ITO layer on PEN increased from $150 \Omega / \square$ to $0.1 \mathrm{M} \Omega / \square$ after 10 to 20 bending cycles. Since the roughness of the ITO after bending is almost comparable to the thickness of the polymer
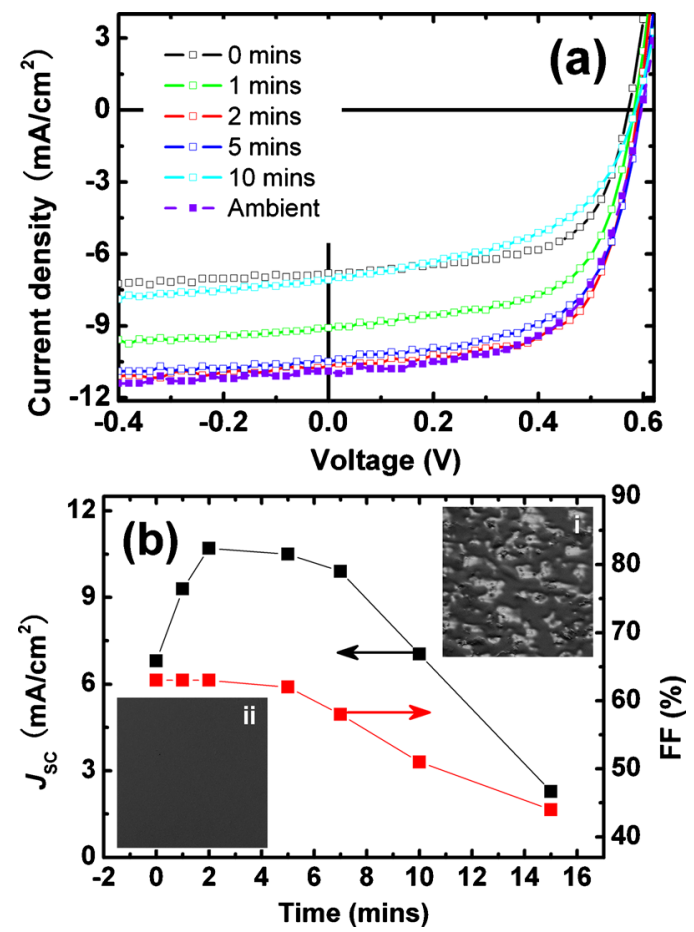

FIG. 3. (Color online) (a) $I-V$ curves of laminated OPVs with Ag anode treated by UV-ozone and Ag anode kept in ambient room condition; (b) $J_{\text {sc }}$ and FF versus UV-ozone treating time for laminated device with $\mathrm{Ag}$ anode. Inset shows the scanning electron microscopy images $(50 \mu \mathrm{m} \times 50 \mu \mathrm{m})$ of E-glue film spin coated on pristine Ag (i) and oxidized Ag films (ii).

active layer, it is likely that the conductive ITO fragments penetrate into the soft polymer layers and form current leakage paths during the lamination process.

The improvement of $J_{\text {sc }}$ by using optimized E-glue/Ag anode can be explained by the following three reasons. First, the light reflection from $\mathrm{Ag}$ anode film increases the light harvesting of the solar cells. Second, the Ag anode collects charges more efficiently than ITO anode. The sheet resistance of the ITO layer on the PEN substrate is $150 \Omega / \square$ (larger if bended). Therefore, there is a considerable voltage drop on the electrode, which leads to a reduced $J_{\text {sc }}$. Third, a thin layer of silver oxide on the Ag surface increases the anode work function and surface energy, which is discussed below. The increase in FF from 55\% (with ITO anode) to $63 \%$ (with $\mathrm{Ag}$ anode) is also attributed to the reduced leakage current and series resistance.

The device with E-glue/Al anode showed poor performance, with a PCE of $1.4 \%$. This can be explained by the formation of a thin insulating alumina layer at the E-glue/Al interface, which typically occurs within seconds of exposure to ambient room conditions. During the study, the Al layer was thermal deposited in a vacuum and then exposed in ambient room conditions for several minutes before spincoating the water based E-glue layer. It was then transferred to a dry $\mathrm{N}_{2}$ glove box for lamination. The oxidation of the $\mathrm{Al}$ layer was confirmed by exposing the Al layer in ambient room conditions for $20 \mathrm{~min}$, which resulted in a lower PCE of $0.3 \%$. On the contrary, no degradation of device was observed with the Ag layer when it is partially oxidized. During the study it was found that the laminated devices showed better performance if the Ag layers were treated by UVozone before the spin-coating of E-glue layer. As shown in Figs. 3(a) and 3(b), The $J_{\text {sc }}$ reaches its maximum after $2 \mathrm{~min}$ 


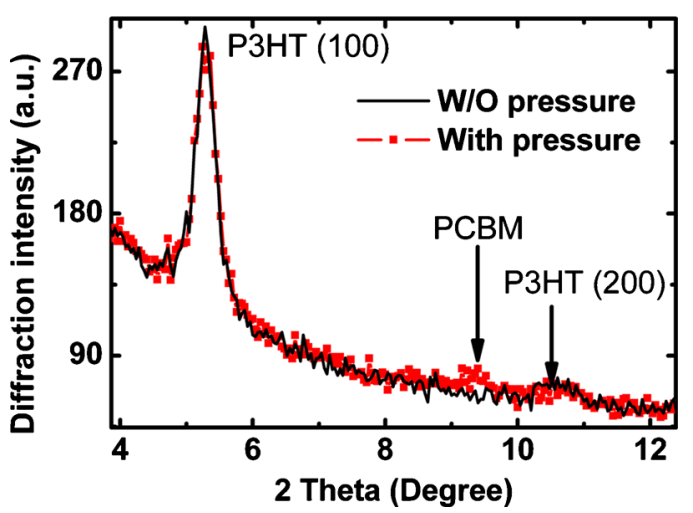

FIG. 4. (Color online) XRD spectra of a P3HT:PCBM film spin coated on a $\mathrm{ITO} / \mathrm{Cs}_{2} \mathrm{CO}_{3}$ substrate with and without a pressure treatment at $100{ }^{\circ} \mathrm{C}$.

of UV-ozone treatment. During the first 5 min of UV-ozone treatment the FF remains about $63 \%$, but after the first $5 \mathrm{~min}$ treatment the FF decreased gradually. Meanwhile the $V_{\text {oc }}$ also increased slightly from around $0.57 \mathrm{~V}$ to around $0.59 \mathrm{~V}$. It should be mentioned that the best E-glue/Ag anode laminated device shown in Fig. 2 has been treated by UV-ozone. The improvement of device performance with the UV-ozone treated Ag layers can be explained by the enhanced surface energy and work function. ${ }^{13,14}$ The scanning electron microscopy images of the E-glue/Ag anode without (i) and with (ii) UV-ozone treatment are shown in the inset of Fig. 3(b). The coverage of E-glue on the $\mathrm{Ag}$ layer increases from roughly $70 \%$ to $100 \%$ with the UV-ozone treatment. This is due to the enhanced surface energy of $\mathrm{Ag}$ surface, which then maximizes the effective device working areas. The UVozone treatment is expected to oxidize the thin layer of silver to silver oxide $\left(\mathrm{AgO}_{\mathrm{x}}\right)$. The work function of $\mathrm{AgO}_{\mathrm{x}}$ layer is reported to be $0.4 \mathrm{eV}$ higher than that of the $\mathrm{Ag}$ layer. ${ }^{14} \mathrm{An}$ ultrathin $\mathrm{AgO}_{\mathrm{x}}$ layer between the $\mathrm{E}$-glue and $\mathrm{Ag}$ anodes may form energy intermediate levels, significantly increasing the charge collection efficiency. ${ }^{13}$ For the devices with UVozone treatment times longer than $2 \mathrm{~min}$, the insulating $\mathrm{AgO}_{\mathrm{x}}$ layers begin to block the charges, causing the reduction in $J_{\mathrm{sc}}$ and FF.

In manufacturing processes, the $\mathrm{Ag}$ layer might be exposed to ambient room conditions and partially oxidized. To test whether this exposure would change the $\mathrm{Ag}$ anode and hence the device performance, the Ag films were intentionally exposed in air for $40 \mathrm{~h}$ before UV-ozone treatment. The result is also listed in Fig. 3(a) which shows the partial oxidization of the Ag film in ambient room conditions would not degrade the laminated devices.

To test whether the lamination process changes the morphology of the P3HT:PCBM film, the x-ray diffraction (XRD) spectra of the samples with and without a laminating treatment were compared (Fig. 4). The intensity of the P3HT crystal $(100)$ peak $\left(2 \theta \approx 5.3^{\circ}\right)$ is nearly unchanged, indicat- ing the crystalline structure of P3HT remain unchanged. A PCBM peak at $2 \theta \approx 9.3^{\circ}$ was observed after the laminating treatment, ${ }^{15}$ which indicates the PCBM crystallizes during the lamination process. The PCBM crystallization under pressure might be caused by the PCBM diffusion through the gaps that open up between P3HT crystalline grains. ${ }^{16}$ This study proposes the highly crystalline P3HT:PCBM film induced by the lamination process led to a higher $J_{\mathrm{sc}}$ and FF, although other improving contributions cannot be ignored, such as avoiding the damage of the P3HT:PCBM layer caused by the thermally deposited metal anode and forming a high-surface-area-structure by the penetration of the highly conductive E-glue into the P3HT:PCBM layer during the lamination. ${ }^{6}$

In summary, this study reported the fabrication of high efficiency OPVs with Ag as anode by a vacuum free lamination process. The optimized lamination devices have a PCE of $4.0 \%$ which is the same with that of regular devices fabricated by thermal evaporation. The $\mathrm{Ag}$ layer is stable in ambient room conditions before laminating, which satisfies the requirements of manufacturing. Another advantage to using silver is that the partial oxidization actually increases the efficiency of the devices so that no extra protection is needed during the manufacturing. One interesting finding is the pressure in the lamination process promotes the crystallization of PCBM, which provides another way to modify the active layer morphology in optimizing the device performance.

${ }^{1}$ M. Nakamura, C. Yang, E. Zhou, K. Tajima, and K. Hashimoto, ACS Appl. Mater. Interfaces 1, 2703 (2009).

${ }^{2}$ M. Nakamura, C. H. Yang, K. Tajima, and K. Hashimoto, Sol. Energy Mater. Sol. Cells 93, 1681 (2009).

${ }^{3}$ J. B. Kim, S. Lee, M. F. Toney, Z. H. Chen, A. Facchetti, Y. S. Kim, and Y. L. Loo, Chem. Mater. 22, 4931 (2010).

${ }^{4}$ W. Gaynor, J. Y. Lee, and P. Peumans, ACS Nano 4, 30 (2010).

${ }^{5}$ J. Ouyang and Y. Yang, Adv. Mater. 18, 2141 (2006).

${ }^{6}$ J. S. Huang, G. Li, and Y. Yang, Adv. Mater. 20, 415 (2008).

${ }^{7}$ V. Shrotriya, G. Li, Y. Yao, C. W. Chu, and Y. Yang, Appl. Phys. Lett. 88, 073508 (2006).

${ }^{8}$ V. Shrotriya, G. Li, Y. Yao, T. Moriarty, K. Emery, and Y. Yang, Adv. Funct. Mater. 16, 2016 (2006).

${ }^{9}$ N. Li, B. E. Lassiter, R. R. Lunt, G. Wei, and S. R. Forrest, Appl. Phys. Lett. 94, 023307 (2009).

${ }^{10} \mathrm{~K}$. Vandewal, K. Tvingstedt, A. Gadisa, O. Inganäs, and J. V. Manca, Nature Mater. 8, 904 (2009).

${ }^{11}$ W. J. Potscavage, S. Yoo, and B. Kippelen, Appl. Phys. Lett. 93, 193308 (2008).

${ }^{12}$ G. F. Wang, X. M. Tao, and R. X. Wang, Nanotechnology 19, 145201 (2008).

${ }^{13}$ W. J. Yoon and P. R. Berger, Appl. Phys. Lett. 92, 013306 (2008).

${ }^{14}$ H. W. Choi, S. Y. Kim, K. B. Kim, Y. H. Tak, and J. L. Lee, Appl. Phys. Lett. 86, 012104 (2005).

${ }^{15}$ T. Erb, U. Zhokhavets, G. Gobsch, S. Raleva, B. Stühn, P. Schilinsky, C. Waldauf, and C. J. Brabec, Adv. Funct. Mater. 15, 1193 (2005).

${ }^{16}$ M. Campoy-Quiles, T. Ferenczi, T. Agostinelli, P. G. Etchegoin, Y. Kim, T. D. Anthopoulos, P. N. Stavrinou, D. D. C. Bradley, and J. Nelson, Nature Mater. 7, 158 (2008). 